\title{
Validación de contenido y adaptación de la escala de sentido de coherencia 29 para la población colombiana
}

\author{
Content validation and adaptation of the 29-item Sense of Coherence scale for the \\ Colombian population
}
Validação de conteúdo e adaptação da escala senso de coerência (soc-29) para a população colombiana

\begin{abstract}
Natalia Sánchez Palacio'; Consuelo Vélez Álvarez²; Diana Paola Betancurth Loaiza
1 Enfermera, magíster en Salud Pública. Universidad de Caldas, Manizales, Colombia. natalia.sanchez@ucaldas.edu.co. ORcid: https:// orcid.org/0000-0002-3405-5144

2 Enfermera, doctora en Salud Pública. Universidad de Caldas, Manizales, Colombia. consuelo.velez@ucaldas.edu.co. ORcid: https:// orcid.org/0000-0001-7274-7304

3 Enfermera, doctora en Salud Pública. Universidad de Caldas, Manizales, Colombia. diana.betancurth@ucaldas.edu.co. ORCID: https:// orcid.org/0000-0001-7620-2336
\end{abstract}

Recibido: 22/05/2020. Aprobado: 09/06/2021. Publicado: 14/07/2021

Sánchez-Palacio N, Vélez-Álvarez C, Betancourth-Loaiza DP. Validación de contenido y adaptación de la escala de sentido de coherencia 29 para la población colombiana. Rev. Fac. Nac. Salud Pública. 2021;39(3):e342827. Dor: https://doi.org/10.17533/ udea.rfnsp.e342827

\section{Resumen}

Objetivo: Validar el contenido de la escala de sentido de coherencia 29 para la población colombiana, la cual mide la capacidad de las personas para aprovechar los recursos materiales e inmateriales que dispone para enfrentar los estímulos presentes a lo largo de la vida. Metodología: Investigación del tipo validación de instrumentos, realizada bajo la técnica de juicio de expertos. El procedimiento contiene cinco pasos: preparación de instrucciones y planilla, selección de expertos, explicación del contexto, discusión y establecimiento de acuerdos. Los criterios de calidad valorados para la validación de contenido fueron: "pertinencia", "relevancia", "utilidad", "suficiencia", "claridad" y "apariencia". Se contó con el consentimiento informado de los participantes. Resultados. La escala de sentido de coherencia fue adaptada acorde con ciertos términos desde el punto de vista semántico y sintáctico, y según las pruebas realizadas, posee buena consistencia interna, con un Alfa de Cronbach de 0,838 y un valor global del índice de validez de contenido considerado como esencial de 0,83. Conclusiones. Los hallazgos cuantitativos y cualitativos del proceso de validación confirman que la escala en versión español puede ser aplicada en población colombiana, con el fin de medir el sentido de coherencia, entendido como la capacidad de aprovechar los recursos para enfrentar los estímulos.

--Palabras clave: Estudio de validación, instrumentos, revisión por pares, salud pública, sentido de coherencia. 


\begin{abstract}
Objective: To validate, for the Colombian population, the content of the 29-item Sense of Coherence scale, which measures the ability of people to take advantage of material and immaterial resources available for them to face the stimuli present throughout life. Methodology: Instrument validation research, carried out under the expert judgment technique. The procedure contains five steps: preparation of instructions and worksheet, selection of experts, explanation of the context, discussion, and establishment of agreements. The quality criteria assessed for content validation were: "pertinence," "relevance," "usefulness," "sufficiency," "clarity," and "appearance." Informed consent was obtained from all the participants. Results: The Sense of Coherence
\end{abstract}

scale was adapted according to certain terms from the semantic and syntactic point of view, and according to the tests carried out, it has good internal consistency, with a Cronbach's alpha of 0.838 and a global value of the content validity index considered as essential of 0.83 . Conclusions: The quantitative and qualitative findings of the validation process confirm that the scale version in Spanish can be applied in the Colombian population with the aim of measuring the sense of coherence, understood as the ability to take advantage of resources to face stimuli.

--------Keywords: validation study, instruments, peer review, public health, sense of coherence.

\section{Resumen}

Objetivo: Validar o conteúdo da escala senso de coerência 29 para a população colombiana, a qual mede a capacidade das pessoas para aproveitar os recursos materiais e imateriais disponíveis para enfrentar os estímulos presentes ao longo da vida. Metodologia: Estudo do tipo validação de instrumentos, realizado com a técnica de julgamento de especialistas. O procedimento está composto por cinco passos: preparação de instruções e planilha, seleção de especialistas, explicação do contexto, discussão e estabelecimento de acordos. Os critérios de qualidade analisados para a validação de conteúdo foram: "pertinência", "relevância", "utilidade", "suficiência", "clareza" e "aparência". Contou-se com o consentimento informado dos participantes. Resultados: A escala senso de coerência foi adaptada de acordo com certos termos partindo do ponto de vista semântico e sintático, e de acordo com os testes realizados, possui boa consistência interna, com um Alfa de Cronbach de 0,838 e um valor global de índice de validade de conteúdo considerado como essencial de 0,83. Conclusões: As descobertas quantitativas e qualitativas do processo de validação confirmam que a escala na versão em espanhol pode ser aplicada na população colombiana, com o objetivo de medir o sentido de coerência, entendido como a capacidade de aproveitar os recursos para enfrentar os estímulos.

---------Palavras-chave: Estudo de validação; Instrumentos; Revisão por pares; Saúde pública; Sentido de coerência.

\section{Introducción}

Una de las teorías que buscó fortalecer el nuevo paradigma de salud, en el cual se integran los aspectos físicos, mentales y sociales de las personas, fue el modelo salutogénico propuesto por Aaron Antonovsky en 1979. En esta perspectiva, se critica el enfoque salud-enfermedad y se propone entender la salud como un continuo entre dos polos: el bienestar (salud) y el malestar (enfermedad). Por ende, "la salud no es un estado de equilibrio pasivo, sino, más bien, un proceso inestable, de autorregulación activa y dinámica" [1, p. 14].

El modelo se compone de tres elementos: 1) el centro de atención se ubica en la resolución de problemas y la búsqueda de soluciones; 2) identifica los recursos generales de resistencia, que permiten a las personas percibir su vida como coherente, estructurada y comprensible; y 3) señala un sentido global en los individuos, grupos y poblaciones, el cual ayuda a que, más allá de poseer recursos, se tenga la capacidad para aprovechar- los, es decir, poseer sentido de coherencia (Sense of Coherence, soc) [1,2].

Investigaciones llevadas a cabo en Colombia han abordado el modelo salutogénico y han desarrollado el concepto de soc no solo desde el campo de la promoción de la salud, sino también desde otras perspectivas, como las ambientales, médicas, comparativas, etc. [36]. Estas aproximaciones realizan un aporte de elementos teóricos y metodológicos para futuros estudios, y al profundizar en algunas de ellas, se evidencia la necesidad de avanzar en el fortalecimiento de esta concepción - especialmente la de soc- como un abordaje trascendental para la salud.

Antonovsky introdujo la escala denominada "Escala de orientación hacia la vida" (Orientation to Life Questionnaire, OLQ), también llamada "Escala de sentido de coherencia" (escala soc), compuesta por tres dominios cognitivos (la comprensibilidad, la manejabilidad y la significatividad) y dos versiones: una conformada por 29 ítems originalmente y otra reducida a 
13 ítems [1]. Con las aplicaciones de la escala, se identifica que el soc alto se asocia con una salud buena y se convierte en un factor protector ante la ansiedad, la depresión, el estrés, el agotamiento y la desesperanza, fuera de que ayuda al optimismo, la fortaleza, el autocontrol y la capacidad de afrontamiento [2].

La escala soc, en sus dos versiones, ha sido validada en diversas investigaciones, especialmente en España [1,2,7-10]. Asimismo, fue traducida a diferentes idiomas y aplicada a distintas poblaciones a nivel mundial, entre las que se destacan adolescentes, jóvenes, adultos, adultos mayores y poblaciones con alguna condición de enfermedad, como esclerosis múltiple, diabetes, enfermedad cardiovascular y enfermedad pulmonar obstructiva crónica. Estos datos la han llevado a ser calificada como válida y fiable.

A nivel de Latinoamérica, en países como México, Perú y Ecuador [8-10] se han realizado varios estudios de validación del instrumento, pero no se cuenta con una validación en Colombia, que permita valorar el nivel de soc. Por ello, el objetivo de este artículo es validar el contenido de la escala soc-29 para la población colombiana.

\section{Metodología}

Esta investigación es del tipo validación de instrumentos, realizada bajo la técnica de juicio de expertos, con la cual se pretende llevar fenómenos no observables de manera directa a constructos observables con indicadores, a través de procesos de medición [11].

La construcción de instrumentos que recojan la mayoría de propiedades de rigurosidad científica deberían ser transversales en todo proceso investigativo; esto favorece la consecución de resultados válidos y confiables [11].

\section{Características de la escala de sentido de coherencia 29}

soc es la capacidad del sujeto de aprovechar los capitales que tiene y hacer frente a las situaciones que se le presentan durante su vida. El desarrollo del sentido de coherencia permite brindar fortaleza, autocontrol y capacidad de afrontamiento, y ver la vida de manera coherente, estructurada, comprensible, manejable y significativa [12-14].

La escala consta de 29 ítems, por medio de los cuales se evalúan tres dominios cognitivos $[1,2,13]$ :

1. La comprensibilidad: definida como la potencialidad de los sujetos para comprender cómo está organizada su vida y cómo se sitúan frente al mundo. Es el componente cognoscitivo de constructo que favorece la capacidad para interactuar con el entorno, entender las situaciones de los otros y controlar los propios pensamientos y emociones (11 ítems).

2. La manejabilidad: entendida como la posibilidad que configuran las personas para manejar sus vidas.
Es el componente instrumental de la escala, pues mide el grado en que un individuo entiende que los recursos que necesita para su supervivencia están a su disposición, independiente de poseer autocontrol o depender del entorno (10 ítems).

3. La significatividad: concebida como la manera en que las personas sienten que su vida se orienta hacia metas concretas. Evalúa el aspecto motivacional de la escala, y a los obstáculos, como retos valiosos de la vida en las personas ( 8 ítems).

\section{Procedimiento}

Se presentan los cinco pasos llevados a cabo para el proceso de validación de contenido por juicio de expertos, los cuales son tenidos en cuenta para mostrar los resultados. Se retomó el modelo propuesto por Skjong y Wentworht (2000) y Arquer (1995) (citados en Escobar y Cuervo [15, p. 29]):

1. Preparación de instrucciones y planillas. La escala se organizó en una planilla que contenía los ítems del instrumento, lo que permitió a cada experto valorarlos en una escala de 1 a 4 , donde 1 es totalmente en desacuerdo (ítem innecesario); 2, bajo nivel de acuerdo (ítem útil, pero prescindible); 3 , moderado nivel de acuerdo (ítem útil), y 4 , totalmente de acuerdo (ítem esencial) [16]. Los criterios de calidad valorados fueron "pertinencia", "relevancia", "utilidad", "suficiencia", "claridad" y "apariencia". A cada uno de los expertos se le remitió la guía de evaluación, con las instrucciones.

2. Selección de expertos. Se realizó, en primer lugar, el análisis de los perfiles de 99 expertos en el área de salud pública y afines, inscritos en las bases de datos del Ministerio de Ciencia, Tecnología e Innovación (Minciencias), teniendo en cuenta la experiencia y la calidad académica valorada según su producción científica, para aportar y retroalimentar el instrumento en sus diferentes ítems, según los criterios de calidad enunciados en el numeral anterior. En total, 42 expertos cumplieron con los siguientes criterios de inclusión:

- Nacionalidad colombiana.

- Conocimiento y experiencia investigativa en el área de salud pública y afines.

- Reconocimiento académico en el área, mediante el análisis de la calidad de las publicaciones, realizado por los autores.

- Nivel de formación mínimo de maestría.

- Mínimo 5 años de experiencia académica en el área.

- Producción bibliográfica en temas relacionados con el área en los últimos 2 años.

- Índice citacional H5 mínimo 2 (verificado en Google Scholar), métrica que es utilizada para la evaluación del impacto de la productividad científica de los investigadores. 
- Participación voluntaria en el estudio.

Una vez enviada la invitación por correo electrónico, en la que se precisaban el objetivo y el procedimiento de la fase de validación, se obtuvo respuesta asertiva de 17 expertos y recepción del concepto por parte de 16 .

3. Explicar el contexto. El instrumento fue adaptado al contexto colombiano. Como queda dicho, se resaltó como uno de los criterios de selección de los expertos la nacionalidad, para que cada ítem fuera analizado según las características particulares de la población objetivo y del territorio.

4. Posibilitar la discusión. Una vez interpretados los resultados de la validación de contenido, se incluyeron los nuevos elementos propuestos por los expertos para realizar la adaptación de manera cualitativa.

5. Establecer el acuerdo entre los expertos por medio del cálculo de consistencia. Para el análisis cuantitativo, se usó el software SPSS versión 22, licenciada por la Universidad de Caldas. Se calculó el Alfa de Cronbach por cada una de las dimensiones y para el instrumento en general. Para la valoración final, se tuvo en cuenta la escala de rangos de interpretación de valores presentada por Ruiz Bolívar [17]:

- 0,81-1,00: Muy alta.

- 0,61-0,80: Alta.

- 0,41-0,60: Moderada.

- 0,21-0,40: Baja.

- 0,01-0,20: Muy baja.

De forma complementaria, se llevó a cabo el cálculo del índice de validez de contenido (IVC) propuesto por Lawshe en 1975, con el fin de evaluar los ítems que debían ser mantenidos en la versión validada del instrumento [18]. Para esto, se partió de la evaluación individual de cada ítem, a través de la medición de la razón de validez de contenido (RVC), que determina si un ítem es esencial para evaluar el constructo; es útil, pero prescindible, o si es innecesario. Esta fórmula asigna una puntuación entre $-1 \mathrm{y}+1$, donde un valor igual o mayor que 0,51 es suficiente para dejar el ítem en la versión final del instrumento [11,18-20].

Por último, una vez definidos aquellos elementos que cuentan con valores superiores a los mínimos propuestos por Lawshe, se calcula la media de RVC para obtener el IVC de toda la prueba, cuyo valor debe ser superior a 0,8 [18]. De esta manera, el IVC se manifiesta en la siguiente ecuación:

$$
I V C=\bar{x} R C V
$$

donde:

$$
R V C=\frac{n-N / 2}{N / 2}
$$

$n$ : número de expertos que asignan calificación esencial al ítem, y

$N$ : número total de expertos que evalúan el ítem.

\section{Consideraciones éticas}

Según la Resolución 8430 de 1993 [21] del Ministerio de Salud de Colombia y la Declaración de Helsinki promulgada por la Asociación Médica Mundial de 2000 [22], la investigación se consideró un estudio sin riesgo.

El proyecto "Propiedades psicométricas de la escala de sentido de coherencia (soc-29) en jóvenes universitarios del municipio de Manizales", que dio origen a este artículo, fue aprobado por el Comité de Bioética de la Facultad de Ciencias para la Salud de la Universidad de Caldas, bajo el consecutivo CBCs-003, Acta 001 del 4 de febrero de 2020.

Además, se contó con el consentimiento informado de los participantes y en todas las fases se conservó el anonimato de los expertos.

\section{Resultados}

Los resultados del estudio se presentan y describen en cinco fases, como parte del proceso de validación y adaptación de la escala soc-29 para población colombiana.

1. Preparación de instrucciones y planillas: para esta fase del proceso, se hizo una revisión documental, que permitió identificar diferentes criterios y escalas que pudieran utilizarse en el proceso de juicio de expertos; por decisión de las investigadoras, la planilla seleccionada reunía los criterios de calidad ya enunciados y se presenta en la Tabla 1. Con esta planilla, se buscaba facilitar la valoración de cada ítem y, además, favorecer la posibilidad de incluir observaciones acertadas en el proceso de adaptación al contexto colombiano.

2. Selección de expertos: para ello, se tuvieron en cuenta los criterios de inclusión expresados en la metodología. Es importante resaltar su disponibilidad e interés en aportar al estudio y otras características como: imparcialidad, independencia y experiencia docente e investigadora en el área, en salud pública o afines. Todos los expertos pertenecían a instituciones de educación superior a nivel nacional.

En cuanto al grado de formación, todos tenían maestría y la mayoría eran doctores o estaban en proceso de culminación de doctorado. Quienes eran docentes se encontraban adscritos al escalafón en sus instituciones de educación superior.

Sobre los años de experiencia, lo mínimo fue 10 años y máximo 38 años. El índice de citaciones (H5) osciló entre 2 y 12 (véase Tabla 2).

3. Explicar el contexto: todos los expertos son colombianos, para garantizar que las observaciones fueran acordes con la realidad. Dentro de los principales aspectos sugeridos para lograr la adaptación se destacan: 
Tabla 1. Planilla de evaluación

\begin{tabular}{|c|c|c|c|c|c|c|c|c|c|c|c|c|c|c|c|c|c|c|c|c|c|}
\hline \multirow{2}{*}{$\begin{array}{c}\text { Dimensión } \\
\text { Comprensibilidad }\end{array}$} & \multicolumn{4}{|c|}{ Pertinencia* } & \multicolumn{4}{|c|}{ Relevancia $^{\dagger}$} & \multicolumn{4}{|c|}{ Utilidad ${ }^{\ddagger}$} & \multicolumn{4}{|c|}{ Suficiencia ${ }^{\S}$} & \multicolumn{4}{|c|}{$\begin{array}{c}\text { Claridad y } \\
\text { apariencia }^{* *}\end{array}$} & \multirow[t]{2}{*}{ Observaciones } \\
\hline & 1 & 2 & 3 & 4 & 1 & 2 & 3 & 4 & 1 & 2 & 3 & 4 & 1 & 2 & 3 & 4 & 1 & 2 & 3 & 4 & \\
\hline \multicolumn{22}{|l|}{ Ítems } \\
\hline \multicolumn{22}{|l|}{ Dimensión } \\
\hline Manejabilidad & 1 & 2 & 3 & 4 & 1 & 2 & 3 & 4 & 1 & 2 & 3 & 4 & 1 & 2 & 3 & 4 & 1 & 2 & 3 & 4 & \\
\hline \multicolumn{22}{|l|}{ Ítems } \\
\hline \multicolumn{22}{|l|}{ Dimensión } \\
\hline Significatividad & 1 & 2 & 3 & 4 & 1 & 2 & 3 & 4 & 1 & 2 & 3 & 4 & 1 & 2 & 3 & 4 & 1 & 2 & 3 & 4 & \\
\hline Ítems & & & & & & & & & & & & & & & & & & & & & \\
\hline
\end{tabular}

¿Considera usted que al instrumento le hace falta un nuevo ítem? Sí

No

¿Cuál propondría?

¿Por qué?

* Existe correspondencia entre el contenido de los ítems y la dimensión para el cual es utilizado.

$\uparrow$ El ítem es esencial o importante para la dimensión evaluada.

\$ El ítem tiene la capacidad de servir o de ser aprovechado para la dimensión en la que es utilizado.

$\S$ Los ítems que pertenecen a una misma dimensión bastan para obtener la medición de esta.

** Grado en que el ítem está redactado de forma clara y precisa.

Tabla 2. Perfiles de expertos que validaron el instrumento

\begin{tabular}{|c|c|c|c|c|c|}
\hline Afiliación institucional & Área de experticia & $\begin{array}{c}\text { Máximo nivel académico } \\
\text { alcanzado }\end{array}$ & $\begin{array}{c}\text { Categoría } \\
\text { docente }\end{array}$ & $\begin{array}{c}\text { Años de } \\
\text { experiencia }\end{array}$ & $\begin{array}{c}\text { Índice } \\
\text { H5 }\end{array}$ \\
\hline Universidad de Nariño & $\begin{array}{l}\text { - Salud pública } \\
\text { - Promoción de la salud }\end{array}$ & $\begin{array}{l}\text { - Enfermera } \\
\text { - Magíster en Salud Pública }\end{array}$ & Titular & 40 & 12 \\
\hline $\begin{array}{l}\text { Universidad de } \\
\text { Antioquia }\end{array}$ & $\begin{array}{l}\text {-Bases teóricas y modelos } \\
\text { aplicados en salud } \\
\text { pública } \\
\text { - Políticas públicas y salud }\end{array}$ & $\begin{array}{l}\text { - Fonoaudiólogo } \\
\text { - Especialista en Promoción de } \\
\text { la Salud } \\
\text { - Magíster en Salud Pública } \\
\text { - Doutor em Saude Publica }\end{array}$ & Titular & 25 & 9 \\
\hline $\begin{array}{l}\text { Universidad de } \\
\text { Antioquia }\end{array}$ & $\begin{array}{l}\text { - Salud pública } \\
\text { - Salud mental } \\
\text { - Mujeres } \\
\text { - Comunidades } \\
\text { en condición de } \\
\text { vulnerabilidad }\end{array}$ & $\begin{array}{l}\text { - Psicóloga } \\
\text { - Magíster en Salud Pública } \\
\text { - Candidata a doctora en } \\
\text { Ciencias Sociales, Niñez y } \\
\text { Juventud }\end{array}$ & Asociada & 16 & 8 \\
\hline $\begin{array}{l}\text { Universidad Autónoma } \\
\text { de Manizales }\end{array}$ & - Salud y juventud & $\begin{array}{l}\text { - Odontóloga } \\
\text { - Magíster en Administración en } \\
\text { Salud } \\
\text { - Doctora en Ciencias Sociales, } \\
\text { Niñez y Juventud }\end{array}$ & Titular & 25 & 7 \\
\hline $\begin{array}{l}\text { Universidad de la } \\
\text { Sabana }\end{array}$ & $\begin{array}{l}\text { - Atención primaria } \\
\text { - Salud comunitaria } \\
\text { - Salud pública }\end{array}$ & $\begin{array}{l}\text { - Médico } \\
\text { - Máster en Gobierno y Dirección } \\
\text { Sanitaria } \\
\text { - Máster en Investigación en } \\
\text { Atención Primaria } \\
\text { - Doctor en Investigación Clínica }\end{array}$ & Asociado & 15 & 7 \\
\hline
\end{tabular}




\begin{tabular}{|c|c|c|c|c|c|}
\hline Afiliación institucional & Área de experticia & $\begin{array}{c}\text { Máximo nivel académico } \\
\text { alcanzado }\end{array}$ & $\begin{array}{c}\text { Categoría } \\
\text { docente }\end{array}$ & $\begin{array}{c}\text { Años de } \\
\text { experiencia }\end{array}$ & $\begin{array}{c}\text { Î́ndice } \\
\text { H5 }\end{array}$ \\
\hline Universidad de Caldas & $\begin{array}{l}\text { - Salud laboral } \\
\text { - Salud pública } \\
\text { - Investigación } \\
\text { - Grupo poblacional: } \\
\text { jóvenes }\end{array}$ & $\begin{array}{l}\text { - Enfermera } \\
\text { - Especialista en Salud } \\
\text { Ocupacional } \\
\text { - Magíster en Educación y } \\
\text { Desarrollo Humano } \\
\text { - Doctora en Ciencias Sociales, } \\
\text { Niñez y Juventud }\end{array}$ & Titular & 26 & 7 \\
\hline Universidad de Caldas & $\begin{array}{l}\text { - Atención primaria de } \\
\text { salud } \\
\text { - Promoción de la salud } \\
\text { y prevención de la } \\
\text { enfermedad } \\
\text { - Gestión del conocimiento }\end{array}$ & $\begin{array}{l}\text { - Enfermera } \\
\text { - Magíster en Salud Pública }\end{array}$ & Asistente & 10 & 5 \\
\hline Universidad de Caldas & -Promoción de la salud & $\begin{array}{l}\text { - Enfermera } \\
\text { - Magíster en Investigación y } \\
\text { Tecnología Educativa }\end{array}$ & Asociada & 38 & 5 \\
\hline $\begin{array}{l}\text { Universidad Pedagógica } \\
\text { y Tecnológica de } \\
\text { Colombia }\end{array}$ & $\begin{array}{l}\text { - Universidad promotora } \\
\text { de salud } \\
\text { - Comunidad académica } \\
\text { universitaria }\end{array}$ & $\begin{array}{l}\text { - Psicóloga } \\
\text { - Especialista en Educación } \\
\text { Sexual } \\
\text { - Especialista en Pedagogía } \\
\text { - Magíster en Desarrollo } \\
\text { Educativo y Social } \\
\text { - Doctora en ciencias Sociales, } \\
\text { Niñez y Juventud }\end{array}$ & Asociada & 29 & 5 \\
\hline $\begin{array}{l}\text { Universidad de } \\
\text { Antioquia }\end{array}$ & $\begin{array}{l}\text { - Promoción de la salud } \\
\text { - Promoción de la salud } \\
\text { mental } \\
\text { - Grupo poblacional de } \\
\text { trabajo: niños, jóvenes, } \\
\text { adultos, adultos mayores }\end{array}$ & $\begin{array}{l}\text { - Médica } \\
\text { - Magíster en Salud Pública con } \\
\text { énfasis en Salud Mental }\end{array}$ & No aplica & 37 & 3 \\
\hline $\begin{array}{l}\text { Universidad Antonio } \\
\text { Nariño }\end{array}$ & $\begin{array}{l}\text { - Salud pública } \\
\text { - Salud sexual y } \\
\text { reproductiva }\end{array}$ & $\begin{array}{l}\text { - Enfermero } \\
\text { - Magíster en Enfermería con } \\
\text { énfasis en Gerenci }\end{array}$ & No aplica & 21 & 3 \\
\hline $\begin{array}{l}\text { Universidad } \\
\text { Surcolombiana de Neiva }\end{array}$ & $\begin{array}{l}\text { - Salud de poblaciones } \\
\text { - Salud pública } \\
\text { - Promoción de salud }\end{array}$ & $\begin{array}{l}\text { - Enfermera } \\
\text { - Magíster en Educación y } \\
\text { Desarrollo Comunitario } \\
\text { - Doctora en Ciencias de la } \\
\text { Salud } \\
\text { - Estancia posdoctoral en } \\
\text { Políticas Públicas de Salud }\end{array}$ & Titular & 35 & 3 \\
\hline $\begin{array}{l}\text { Universidad de } \\
\text { Antioquia }\end{array}$ & $\begin{array}{l}\text { - Salud colectiva } \\
\text { - Enfermería comunitaria } \\
\text { - Educación para la salud } \\
\text { - Pedagogía del cuidado }\end{array}$ & $\begin{array}{l}\text { - Enfermera } \\
\text { - Magíster en Salud Colectiva } \\
\text { - Candidata a doctora en } \\
\text { Salud Colectiva, Ambiente y } \\
\text { Sociedad }\end{array}$ & Asistente & 14 & 2 \\
\hline Universidad de Ibagué & $\begin{array}{l}\text { - Promoción de la salud } \\
\text { - Salud mental comunitaria } \\
\text { - Formación de docentes y } \\
\text { pedagogía }\end{array}$ & $\begin{array}{l}\text { - Filósofo } \\
\text { - Psicólogo } \\
\text { - Maestro en Salud Mental } \\
\text { Comunitaria } \\
\text { - Maestro en Educación }\end{array}$ & Asistente & 8 & 2 \\
\hline
\end{tabular}




\begin{tabular}{|c|c|c|c|c|c|}
\hline Afiliación institucional & Área de experticia & $\begin{array}{c}\text { Máximo nivel académico } \\
\text { alcanzado }\end{array}$ & $\begin{array}{c}\text { Categoría } \\
\text { docente }\end{array}$ & $\begin{array}{c}\text { Años de } \\
\text { experiencia }\end{array}$ & $\begin{array}{c}\text { Índice } \\
\text { H5 }\end{array}$ \\
\hline $\begin{array}{l}\text { Universidad de los } \\
\text { Llanos }\end{array}$ & $\begin{array}{l}\text { - Salud pública } \\
\text { - Salud familiar } \\
\text { - Salud colectiva }\end{array}$ & $\begin{array}{l}\text { - Enfermera } \\
\text { - Especialista en Salud Familiar } \\
\text { - Máster en Investigación en } \\
\text { Atención Primaria } \\
\text { - Magíster en Desarrollo } \\
\text { Educativo y Social } \\
\text { - Candidata a doctora en } \\
\text { Ciencias Sociales, Niñez y } \\
\text { Juventud }\end{array}$ & Asociada & 26 & 2 \\
\hline Universidad del Valle & $\begin{array}{l}\text { - Salud pública } \\
\text { - Salud sexual reproductiva } \\
\text { - Bienestar espiritual } \\
\text { - Desarrollo disciplinar } \\
\text { - Validación de } \\
\text { instrumentos }\end{array}$ & $\begin{array}{l}\text { - Enfermera } \\
\text { - Especialista en Medicina } \\
\text { Crítica Pediátrica } \\
\text { - Magíster en Ciencias de la } \\
\text { Enfermería } \\
\text { - Doctora en Enfermería }\end{array}$ & Asistente & 29 & 2 \\
\hline
\end{tabular}

- Modificar las frases que se encuentran en negación, para mejorar la interpretación.

- Revisar la traducción de los términos "confuso", "mucho", "profundo", "impresión".

- Analizar las preguntas con rangos de tiempo muy extensos o ambiguos, dado que difícilmente las personas los recuerdan.

- Ajustar la semántica y la sintaxis de algunos ítems.

- Analizar el uso de términos totalizantes, como "siempre", "nunca", "completamente", "llena".

4. Posibilitar la discusión: el análisis permitió evidenciar la importancia de la secuencia lógica del diseño de la escala en función de los ítems de cada dimensión, toda vez que en la prueba original los ítems se presentan sin un orden por dimensión. Se revisaron los ítems y se modificaron según las recomendaciones.
5. Establecer el acuerdo entre los expertos por medio del cálculo de consistencia: para valorar la consistencia interna, se calculó el Alfa de Cronbach por ítem, para cada una de las dimensiones y para toda la escala. Nótese, en la Tabla 3, cómo, según los parámetros establecidos por Ruiz Bolívar [17] para todas las dimensiones, el valor del coeficiente se ubicó en alto y muy alto, y el total de la escala se ubicó como muy alto.

En la Tabla 4 puede observarse el resultado del cálculo de la RVC y el IVC de la escala soc versión 29 ítems. En esta se nota que todos los ítems del instrumento obtuvieron un valor superior a 0,51 , lo que indica que deben ser incluidos en la versión final del instrumento validado. De igual modo, se encuentra un IVC global de la escala, con un valor 0,83 (IC $95 \%$ : 0,60;0,99), lo que confirma la esencialidad del instrumento.

Se anexa la versión validada y adaptada del instrumento para Colombia.

Tabla 3. Alfa de Cronbach

\begin{tabular}{lc}
\hline \multicolumn{1}{c}{ Escala } & Alfa de Cronbach \\
\hline Dimensión comprensibilidad & 0,83 \\
Dimensión manejabilidad & 0,81 \\
Dimensión significatividad & 0,70 \\
Escala soc-29 & 0,84 \\
\hline
\end{tabular}


Tabla 4. Índice de validez de contenido

\begin{tabular}{|c|c|c|c|c|c|c|}
\hline \multicolumn{2}{|c|}{ Dimensión comprensibilidad } & \multicolumn{2}{|c|}{ Dimensión manejabilidad } & \multicolumn{2}{|c|}{ Dimensión significatividad } & \multirow[t]{2}{*}{ Ivc escala soc } \\
\hline Ítem & RVC & Ítem & RVC & Ítem & RVC & \\
\hline 1 & 0,88 & 1 & 1 & 1 & 0,81 & \multirow{11}{*}{$\begin{array}{c}0,83 \\
(\text { Ic } 95 \%: 0,60 ; 0,99)\end{array}$} \\
\hline 2 & 0,56 & 2 & 0,56 & 2 & 0,75 & \\
\hline 3 & 0,88 & 3 & 0,88 & 3 & 0,93 & \\
\hline 4 & 0,62 & 4 & 0,81 & 4 & 0,93 & \\
\hline 5 & 1 & 5 & 0,88 & 5 & 1 & \\
\hline 6 & 0,69 & 6 & 0,88 & 6 & 0,75 & \\
\hline 7 & 0,69 & 7 & 1 & 7 & 1 & \\
\hline 8 & 0,75 & 8 & 0,81 & \multirow{4}{*}{8} & \multirow{4}{*}{0,81} & \\
\hline 9 & 0,81 & 9 & 0,88 & & & \\
\hline 10 & 0,81 & \multirow{2}{*}{10} & \multirow{2}{*}{0,93} & & & \\
\hline 11 & 0,62 & & & & & \\
\hline
\end{tabular}

RCV: Razón de validez de contenido; IVC: Índice de validez de contenido.

\section{Discusión}

En los resultados de la propuesta de validación de la escala soc-29, los dominios cognitivos de comprensibilidad (parte cognitiva del constructo total), manejabilidad (elemento instrumental) y significatividad (parte motivacional del constructo) otorgan validez de contenido, según el juicio de los expertos en el contexto colombiano. De esta manera, la escala se configura en una orientación que enuncia el grado en que se tiene una sensación de confianza omnipresente, duradera y dinámica en los tres sentidos: 1) los estímulos presentes en el entorno interno y externo al individuo a lo largo de su vida son estructurados, predecibles y explicables (comprensibles); 2) los recursos están disponibles para satisfacer las demandas ambientales derivadas de los estímulos (manejables), y 3) las demandas presentes son retos, los cuales requieren de esfuerzo y compromiso (significativas) $[23,24]$. Lo anterior guarda congruencia con la literatura consultada [25,26].

Para la versión de la escala Soc-29 se destacan las validaciones de contenido realizadas por Velásquez et al., en virtud de que incluyen todos los aspectos descritos en la teoría de Antonovsky; y se deduce que la redacción de los reactivos y la claridad en las opciones de respuesta son algunos de los factores más relevantes [8]. Por el contrario, autores como Möllerberg et al., en 2019, en la validación de contenido de la escala de sentido de coherencia familiar (versión adaptada a las originales), dio a conocer que fue inconsistente según análisis de expertos, hallazgo que podría explicarse por las conexiones conceptuales con la teoría, dado que el soc familiar es más complejo, en relación con el sentido general de coherencia, por la inclusión de los miembros de la familia y su enfoque sistémico [27].

Frente a los criterios de calidad, las categorías de análisis se diferencian según la propuesta de los autores, el objeto y el objetivo de la investigación. Para este caso del instrumento soc-29, la "pertinencia", "relevancia", "utilidad", "suficiencia", "claridad" y "apariencia" fueron las categorías seleccionadas; además, se encontró una relativa solidez. Autores como Galicia et al. utilizan otras categorías, como "coherencia" a cambio de "utilidad" [28] y Cassiani et al., la "pertinencia" y la "adecuación" [29].

Todo instrumento debe presentar una calidad óptima, pues, desde luego, es la manera para asegurar evidencias que sean válidas y fiables; de allí la relevancia del diseño de la planilla para evaluar la validez de contenido por parte de los expertos, según lo afirman Juárez y Tobón [16]. Por su parte, Robles y Rojas entienden que es fundamental prestar atención al momento de elección y categorización de las dimensiones del dominio, a fin de garantizar las inferencias de las observaciones registradas en las mismas [30].

Con respecto a la selección de expertos, la manera como se realizó y los criterios tenidos en cuenta coinciden con lo planteado por Zamora et al., autores que resaltan la importancia de contar, para los procesos de validación de contenido, con especialistas en el tema y lograr conceptos sobre cada uno de los ítems valorados según los criterios de calidad [31]. En este sentido, Bruna et al. confirman la relevancia de tener en cuenta la trayectoria investigativa para seleccionar evaluadores [32]. Al mismo tiempo, con el propósito de lograr que la escala fuera analizada y adaptada al contexto 
colombiano, los expertos seleccionados fueron de nacionalidad colombiana, criterio que también ha sido tenido en cuenta en trabajos como el desarrollado por Botella y Escorihuela [33].

Según las propiedades psicométricas en la versión adaptada a México, el Alfa de Cronbach fue de 0,902 [8]. Como los resultados en la presente investigación arrojaron un Alfa de Cronbach de 0,838, este valor sugiere una muy buena consistencia interna de la escala SOC en su versión de 29 ítems [8]. De igual manera, el IVC encontrado, tanto de cada dimensión como de la escala en general, es considerado como esencial. Por tanto, ambas medidas se convierten en indicadores de la pertinencia de la escala para ser aplicada en población colombiana $[18,34,35]$.

Como limitaciones del estudio, se presentaron algunos retrasos, por la escasa disponibilidad de tiempo de los expertos, dado que es una acción voluntaria; en este sentido, algunos tuvieron que reemplazarse teniendo en cuenta los criterios de inclusión.

\section{Conclusiones}

La selección de expertos cualificados para la evaluación de los ítems de la escala soc-29 contribuyó a tener una evaluación objetiva del instrumento y a adaptarlo de manera óptima para su utilización en el contexto colombiano.

Los hallazgos cuantitativos del proceso de validación de contenido reflejaron un alto nivel de acuerdo de los expertos con respecto a los criterios de calidad evaluados: la pertinencia, relevancia, utilidad, suficiencia, claridad y apariencia del instrumento; estos se soportaron con la buena consistencia interna de la escala, representada en un Alfa de Cronbach de 0,838 y un valor global del IVC de 0,83 , aspectos que permitieron conservar el total de ítems del instrumento y valorar la escala como esencial.

Los resultados de la validez de contenido confirman que la escala soc-29 validada puede ser aplicada en el contexto colombiano para medir el soc, entendido como la capacidad de aprovechar los recursos para enfrentar los estímulos presentes en el curso de vida de las personas.

Para futuras investigaciones, se recomienda evaluar las otras propiedades psicométricas de la escala soc-29, relacionadas con la validez estructural, validez de criterio, de constructo y fiabilidad, de tal manera que se pueda contar con un instrumento adaptado en su estructura factorial, semántica y sintáctica para el contexto colombiano.

\section{Fuente de financiación}

Universidad de Caldas

\section{Conflicto de interés}

Los autores declaran que no hay conflicto de interés

\section{Declaración de responsabilidad}

Los autores declaran que los puntos de vista expresados en el artículo es de nuestra responsabilidad.

\section{Declaración de autoría}

Los autores declaran que contribuyeron en la investigación y elaboración del artículo, y cumplen con los criterios de autoría del International Committee Medical Journals Editors (ICMJE).

\section{Referencias}

1. Rivera F, López A, et al. Propiedades psicométricas de la escala sentido de coherencia (SOC-29) en adolescentes españoles. Rev Psicol da Criança e do Adolesc [internet]. 2011 [citado 2020 may. 19]; (4):11-39. Disponible en: https://idus.us.es/bitstream/ handle/11441/41008/propiedades\%20psicometricas\%20escala. pdf? sequence $=1 \&$ is Allowed $=\mathrm{y}$

2. Guevara MS, Nicolalde TM, Heredia SI. Dimensionalidad y propiedades psicométricas de la escala SOC en estudiantes de la ESPOCH. Rev mktDescubre [internet]. 2017 [citado 2020 may. 19]; 10:84-93. Disponible en: http://revistas.espoch.edu.ec/index.php/ mktdescubre11/article/view/42

3. Vinaccia S, Quiceno JM, Daza M, et al. Factores salutogénicos y patogénicos en padres de niños saludables con dificultad motora. Inf Psicológicos. 2016;16(1):101-15. DoI: https://doi. org/10.18566/infpsicv16n1a06

4. Escobar LN, García GA. El enfoque salutogénico de la psiquiatría. Rev Neuronum [internet]. 2018 [citado 2020 may. 25]; 4(1):1-14. Disponible en: http://eduneuro.com/revista/index.php/ revistaneuronum/article/view/107

5. Garzón MO. Diagnóstico ambiental en salud: desde la corriente salubrista y la corriente ambientalista. Rev ces Salud Pública [internet]. 2016 [citado 2020 may. 19]; 6(2):190-5. https://revistas. ces.edu.co/index.php/ces_salud_publica/article/view/3857/2560

6. Juárez F. El concepto de salud: una explicación sobre su unicidad, multiplicidad y los modelos de salud. Int J Psychol Res. [internet]. 2011 [citado 2020 may. 25];4(1):70-9. Disponible en: https:// www.redalyc.org/pdf/2990/299022819009.pdf

7. Virués-Ortega J, Martínez-Martín P, Del Barrio JL, et al. Validación transcultural de la escala de sentido de coherencia de Antonovsky (olQ-13) en ancianos mayores de 70 años. Med Clin. 2007;128(13):486-92. DoI: https://doi.org/10.1157/13100935

8. Velásquez H, Cárdenas V, Chávez A, et al. Comparación de dos formas de una escala de sentido de coherencia. Rev Intercont Psicol y Educ. [internet]. 2014 [citado 2020 may. 25]; 16(2):51-70. Disponible en: https://www.redalyc.org/pdf/802/80231541004.pdf

9. Lizarbe M, Guillén F, et al. Validación del Cuestionario de Orientación a la Vida (OLQ-13) de Antonovsky en una muestra de estudiantes universitarios en Navarra. An Sist Sanit Navar. 2016;39(2):237-48. DoI: https://doi.org/10.23938/assn.0270 
10. Saravia JC, Iberico C, Yearwood K. Validation of sense of coherence (SOC) 13-item scale in a peruvian sample. J Behav Heal Soc Issues [internet]. 2014 [citado 2020 may. 25];6(2):35-44. Disponible en: https:// www.sciencedirect.com/science/article/pii/S2007078015300031

11. Chiner E. La validez. Alicante: Universidad de Alicante; 2018. pp. 1-13.

12. Vega C, Osuna AF, et al. Validez y confiabilidad de la escala de sentido de coherencia en estudiantes de grado de enfermería de una universidad española. Gac Sanit. 2018;33(4):310-6. DoI: https://doi.org/10.1016/j.gaceta.2018.02.009

13. Lajunen T. Cross-cultural evaluation of Antonovsky's Orientation to Life Questionnaire: Comparison between Australian, Finnish, and Turkish young adults. Psychol Rep. 2019;122(2):731-47. DoI: https://doi.org/10.1177\%2F0033294118765420

14. Mahammadzadeh A, Poursharifi H, Alipour A. Validation of Sense of Coherence (soc) 13-item scale in Iranian sample. Procedia - Soc Behav Sci. 2010; 5:1451-5. DoI: https://doi.org/10.1016/j. sbspro.2010.07.306

15. Escobar J, Cuervo Á. Validez de contenido y juicio de expertos: una aproximación a su utilización. Av en Medición [internet] 2008 [citado 2020 jun. 17]; 6:27-36. Disponible en: http://www. humanas.unal.edu.co/psicometria/files/7113/8574/5708/Articulo3_Juicio_de_expertos_27-36.pdf

16. Juárez LG, Tobón S. Análisis de los elementos implícitos en la validación de contenido de un instrumento de investigación. Rev Espac. [internet]. 2018 [citado 2020 jun. 17]; 39(53):1-23. Disponible en: http://www.revistaespacios.com/cited2017/cited2017-23.pdf

17. Ruiz Bolívar C. Confiabilidad. Programa Interinstitucional Doctorado en Educación. México; 2015. pp. 1-14.

18. Urrutia M, Barrios S, et al. Métodos óptimos para determinar validez de contenido. Rev Cubana Educ Med Super [internet]. 2014 [citado 2020 jun. 17]; 28(3):547-58. Disponible en: http://scielo. sld.cu/pdf/ems/v28n3/ems14314.pdf

19. Sireci S, Faulkner M. Validity evidence based on test content Psicothema. 2014;26(1):100-7. DoI: https://doi.org/10.7334/ psicothema2013.256

20. Ermis H. Establishing content-related validity evidence for assessments in counseling: Application of a sequential mixedmethod approach. Int J Adv Couns. 2018;40(4):387-97. DOI: https://doi.org/10.1007/s10447-018-9332-4

21. Colombia, Ministerio de Salud. Resolución 8430 de 1993, por la cual se establecen las normas científicas, técnicas y administrativas para la investigación en salud (1993 octubre 4).

22. Asociación Médica Mundial. Declaración de Helsinki: principios éticos para las investigaciones médicas en seres humanos [internet] 2000 [citado 2020 jun. 17]. Disponible en: https://www.wma.net/es/ policies-post/declaracion-de-helsinki-de-la-amm-principios-eticospara-las-investigaciones-medicas-en-seres-humanos/

23. Escobar-Castellanos B, Cid P, et al. Estilo de vida promotor de salud y sentido de coherencia en adultos jóvenes universitarios. Hacia. Prom. Salud. 2019;24(2):107-22. DoI: https://doi. org/10.17151/hpsal.2019.24.2.9
24. Braun-Lewensohn O, Sagy S. Salutogénesis and culture: Personal and community sense of coherence among adolescents belonging to three different cultural groups. Int Rev Psychiatry. 2015;23(6):53341. DoI: https://doi.org/10.3109/09540261.2011.637905

25. Savolainen J, Suiminen L, et al. Ethnic disparities in oral health related quality of life among adults in London, England. Community Dent Health 2017;34(2):9-14. DoI: https://doi.org/10.1922/ cdh_4067abderlrahim06

26. Endo S, Kanou H, Oishi K. Sports activities and sense of coherence (SOC) among college students. Int J Sport Heal Sci. 2012;10:111. DoI: https://doi.org/10.5432/ijshs.201114

27. Möllerberg ML, Årestedt K, Sandgren A, et al. Adaptation and psychometric evaluation of the short version of Family Sense of Coherence Scale in a sample of persons with cancer in the palliative stage and their family members. Palliat Support Care. 2019;18(1):1-9. DoI: https://doi.org/10.1017/s1478951519000592

28. Galicia LA, Balderrama JA, Navarro RE. Validez de contenido por juicio de expertos: propuesta de una herramienta virtual. Apert. 2017; 9(2):42-53. DoI: http://dx.doi.org/10.32870/Ap.v9n2.993

29. Cassiani CA, Pérez E, Vargas MC, et al. Validez de apariencia y adaptación de la escala PHQ-9 para la detección de sintomatología depresiva en universitarios de ciencias de la salud de Cartagena (Colombia). Salud Uninorte. 2018;34(1):75-87. DoI: http://dx.doi. org/10.14482/sun.34.1.9154

30. Robles P, Rojas M. La validación por juicio de expertos: dos investigaciones cualitativas en lingüística aplicada. Revista Nebrija de Lingüística Aplicada a la Enseñanza de las Lenguas [internet]. 2015 [citado 2020 jun. 17]; (18):15-21. Disponible en: https://www.nebrija.com/revista-linguistica/la-validacion-por-juicio-de-expertosdos-investigaciones-cualitativas-en-linguistica-aplicada.html

31. Zamora M, Serrano F, Martínez M. Validez de contenido del modelo didáctico P-VIRC (preguntar-ver, interpretar, recorrer, contar) mediante el juicio de expertos. Form Univ. 2020;13(3):43-54. Dor: https://dx.doi.org/10.4067/S0718-50062020000300043

32. Bruna CE, Villarroel VA, et al. Experiencia de diseño y uso de una rúbrica para evaluar informes de laboratorio en formato publicación científica. Form Univ. 2019;12(2):17-28. DoI: http://dx.doi. org/10.4067/S0718-50062019000200017

33. Botella AM, Escorihuela G. Diseño y validación de un cuestionario para conocer la praxis docente del profesorado de flauta travesera en centros superiores de la comunidad valenciana. Rev Comun de la SEECI. 2017; 221(42):1-13. DOI: https://doi.org/10.15198/ seeci.2017.42.1-13

34. Kennedy L, Kichler E, Seabrook J, et al. Validity and reliability of a food skills questionnaire. Nutr Educ Behav. 2019;51(7):857-64. DOI: http://dx.doi.org/10.1016/j.jneb.2019.02.003

35. Mehmannavazan M, Hosseini M, Vartanoosian J, et al. Translation, cultural adaptation and preliminary psychometric evaluation of the "Family Management Measure" among Iranian families with a child with a chronic disease. Electron Physician. 2018;10(6):694250. DoI: https://dx.doi.org/10.19082\%2F6942 


\section{Anexo. Escala soc-29 validada para población colombiana}

1 Cuando habla con las personas, ¿tiene la sensación de que no le comprenden lo que dice?

\begin{tabular}{|l|l|l|l|l|l|l|l|l|}
\hline Nunca lo siento así & 1 & 2 & 3 & 4 & 5 & 6 & 7 & Siempre lo siento así
\end{tabular}

2 En el pasado, cuando usted tuvo que hacer algo que dependía de la cooperación con otros, pensó que:

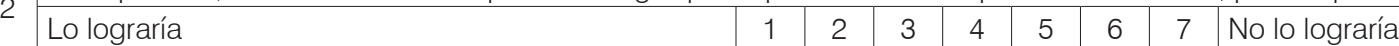

Piense en las personas con las que tiene contacto diario, pero sin considerar a las más cercanas. ¿Qué tanto conoce a esas

3 personas?

\begin{tabular}{|l|l|l|l|l|l|l|l|l}
\hline No las conoce en absoluto & 1 & 2 & 3 & 4 & 5 & 6 & 7 & Las conoce muy bien
\end{tabular}

$4 \quad$ ¿Con qué frecuencia piensa que no le importan las cosas que pasan a su alrededor?

\begin{tabular}{|l|l|l|l|l|l|l|l|l}
\hline Nunca o casi nunca & 1 & 2 & 3 & 4 & 5 & 6 & 7 & Con mucha frecuencia \\
\hline
\end{tabular}

5 ¿Alguna vez en el pasado le ha sorprendido el comportamiento de alguien a quien pensaba conocer muy bien?

\begin{tabular}{l|l|l|l|l|l|l|l|l|l|}
5 & Nunca & 1 & 2 & 3 & 4 & 5 & 6 & 7 & Siempre \\
\hline
\end{tabular}

\begin{tabular}{|l|l|l|l|l|l|l|l|l|l|}
\hline \multirow{2}{*}{6} & ¿Alguna vez se ha decepcionado de la gente en que confiaba? \\
\cline { 2 - 10 } & Nunca & 1 & 2 & 3 & 4 & 5 & 6 & 7 & Siempre \\
\hline
\end{tabular}

\begin{tabular}{|l|l|l|l|l|l|l|l|l|l|}
\hline \multirow{2}{*}{7} & Para usted la vida: & 1 & 2 & 3 & 4 & 5 & 6 & 7 & Es completamente rutinaria \\
\cline { 2 - 8 } & Está llena de interés &
\end{tabular}

\begin{tabular}{|l|l|l|l|l|l|l|l|l|l|}
\hline \multirow{3}{*}{8} & Hasta ahora su vida ha tenido: & 1 & 2 & 3 & 4 & 5 & 6 & 7 & Metas y propósitos muy claros \\
\cline { 2 - 9 } & Metas poco claras
\end{tabular}

\begin{tabular}{|l|l|l|l|l|l|l|l|l|l|}
\hline \multirow{3}{*}{9} & ¿Qué tan seguido piensa que recibe un trato injusto por parte de los demás? \\
\cline { 2 - 9 } & Muy seguido & 1 & 2 & 3 & 4 & 5 & 6 & 7 & Nunca o casi nunca \\
\hline
\end{tabular}

\begin{tabular}{|l|l|l|l|l|l|l|l|l|l|}
\hline \multirow{2}{*}{10} & En los últimos diez años, su vida ha sido: \\
\cline { 2 - 9 } & Llena de cambios sin que sepa qué pasará después & 1 & 2 & 3 & 4 & 5 & 6 & 7 & Totalmente consistente y clara \\
\hline
\end{tabular}

\begin{tabular}{|l|l|l|l|l|l|l|l|l|l|}
\hline \multirow{2}{*}{11} & La mayoría de las cosas que usted hará en el futuro probablemente serán: \\
\cline { 2 - 10 } & Totalmente fascinantes & 1 & 2 & 3 & 4 & 5 & 6 & 7 & Totalmente aburridas \\
\hline
\end{tabular}

\begin{tabular}{|l|l|l|l|l|l|l|l|l|l|}
\hline \multirow{2}{*}{12} & ¿Qué tan seguido se encuentra en una situación en la que no sabe qué hacer? \\
\cline { 2 - 8 } & Muy seguido & 1 & 2 & 3 & 4 & 5 & 6 & 7 & Nunca o casi nunca \\
\hline
\end{tabular}

\begin{tabular}{|l|l|l|l|l|l|l|l|l|l|}
\hline \multirow{2}{*}{13} & ¿Cómo describiría la forma en que usted ve la vida? \\
\cline { 2 - 9 } & Siempre se puede encontrar una solución & 1 & 2 & 3 & 4 & 5 & 6 & 7 & No hay solución a las cosas \\
\hline
\end{tabular}

\begin{tabular}{|l|l|l|l|l|l|l|l|l|l|}
\hline \multirow{2}{*}{14} & Cuando piensa sobre su vida, con frecuencia: \\
\cline { 2 - 8 } & Siente lo bueno que es estar vivo & 1 & 2 & 3 & 4 & 5 & 6 & 7 & Se pregunta para qué vive \\
\hline
\end{tabular}

\begin{tabular}{|l|l|l|l|l|l|l|l|l|l|}
\hline \multirow{2}{*}{15} & Cuando enfrenta un problema, encontrar una solución es: \\
\cline { 2 - 9 } & Siempre confuso y difícil & 1 & 2 & 3 & 4 & 5 & 6 & 7 & Siempre claro y fácil \\
\hline
\end{tabular}

\begin{tabular}{|l|l|l|l|l|l|l|l|l|l|}
\hline \multirow{3}{*}{16} & Hacer lo que hace a diario es: \\
\cline { 2 - 8 } & Siempre fuente de placer y satisfacción & 1 & 2 & 3 & 4 & 5 & 6 & 7 & $\begin{array}{l}\text { Siempre una fuente de dolor y } \\
\text { aburrimiento }\end{array}$ \\
\hline
\end{tabular}

\begin{tabular}{|l|l|l|l|l|l|l|l|l|l|}
\hline \multirow{2}{*}{17} & Su vida en el futuro probablemente será: \\
\cline { 2 - 9 } & Llena de cambios, sin saber qué pasará después & 1 & 2 & 3 & 4 & 5 & 6 & 7 & Totalmente consistente y clara \\
\hline
\end{tabular}

\begin{tabular}{|l|l|l|l|l|l|l|l|l|l|}
\hline \multirow{3}{*}{18} & Cuando algo desagradable sucedió en el pasado, su respuesta fue: \\
\cline { 2 - 8 } & Preocuparse al máximo por ello & 1 & 2 & 3 & 4 & 5 & 6 & 7 & $\begin{array}{l}\text { Decir: "bueno, ya pasó, tengo que } \\
\text { vivir con ello y seguir adelante" }\end{array}$ \\
\hline
\end{tabular}

\begin{tabular}{|l|l}
\hline \multirow{2}{*}{19} & ¿Tiene ideas y sentimientos confusos? \\
\cline { 2 - 2 } & Con mucha frecuencia
\end{tabular}

\begin{tabular}{l|l|l|l|l|l|l|l}
1 & 2 & 3 & 4 & 5 & 6 & 7 & Nunca o casi nunca
\end{tabular} 


\begin{tabular}{|l|l|l|l|l|l|l|l|l|l|}
\hline & \multicolumn{9}{l}{ Cuando usted realiza algo que lo hace sentir bien, piensa que más tarde: } \\
\cline { 2 - 7 } & Seguro seguirá sintiéndose bien & 1 & 2 & 3 & 4 & 5 & 6 & 7 & $\begin{array}{l}\text { Seguro sucederá algo que eche a } \\
\text { perder lo bien que se siente }\end{array}$ \\
\hline
\end{tabular}

\begin{tabular}{|l|l|l|l|l|l|l|l|l|l|}
\hline \multirow{2}{*}{21} & ¿Le ha sucedido que tiene alguna emoción que preferiría no sentir? \\
\cline { 2 - 10 } & Con mucha frecuencia & 1 & 2 & 3 & 4 & 5 & 6 & 7 & Nunca o casi nunca \\
\hline
\end{tabular}

\begin{tabular}{|l|l|l|l|l|l|l|l|l|l|}
\hline 22 & Usted presiente que su vida personal en el futuro: \\
\cline { 2 - 7 } & No tendrá ningún significado ni propósito & 1 & 2 & 3 & 4 & 5 & 6 & 7 & $\begin{array}{l}\text { Estará llena de significado y } \\
\text { propósito }\end{array}$ \\
\hline
\end{tabular}

\begin{tabular}{|l|l|l|l|l|l|l|l|l|l|}
\hline \multirow{2}{*}{23} & ¿Cree que en el futuro siempre habrá personas con las que podrá contar? \\
\cline { 2 - 8 } & Está seguro que sí habrá & 1 & 2 & 3 & 4 & 5 & 6 & 7 & Duda que las haya \\
\hline
\end{tabular}

\begin{tabular}{|l|l|l|l|l|l|l|l|l|l|}
\hline \multirow{2}{*}{24} & ¿Con qué frecuencia siente que no tiene certeza de qué es lo que va a pasar en su vida? \\
\cline { 2 - 9 } & Con mucha frecuencia & 1 & 2 & 3 & 4 & 5 & 6 & 7 & Nunca o casi nunca \\
\hline
\end{tabular}

\begin{tabular}{|l|l|l|l|l|l|l|l|l|l|}
\hline 25 & \multicolumn{2}{l}{$\begin{array}{l}\text { Mucha gente -incluso aquella de carácter fuerte-a veces siente que fracasa en ciertas situaciones. ¿Con cuánta frecuencia } \\
\text { se ha sentido así en el pasado? }\end{array}$} \\
\cline { 2 - 9 } & Nunca & 1 & 2 & 3 & 4 & 5 & 6 & 7 & Con mucha frecuencia \\
\hline
\end{tabular}

\begin{tabular}{|l|l|l|l|l|l|l|l|l|l|}
\hline \multirow{2}{*}{26} & luando algo pasa, por lo general, se ha dado cuenta de que: \\
\cline { 2 - 9 } & Exageró o minimizó su importancia & 1 & 2 & 3 & 4 & 5 & 6 & 7 & $\begin{array}{l}\text { Tomó las cosas en su debida } \\
\text { proporción }\end{array}$ \\
\hline
\end{tabular}

\begin{tabular}{|l|l|l|l|l|l|l|l|l|l|}
\hline 27 & $\begin{array}{l}\text { Cuando piensa en las dificultades que probablemente tendrá que enfrentar en aspectos importantes de su vida, tiene la } \\
\text { impresión de que: }\end{array}$ \\
\cline { 2 - 6 } & Siempre tendrá éxito para superar las dificultades & 1 & 2 & 3 & 4 & 5 & 6 & 7 & $\begin{array}{l}\text { No tendra para superar las } \\
\text { dificultades }\end{array}$ \\
\hline
\end{tabular}

\begin{tabular}{|c|c|c|c|c|c|c|c|c|c|}
\hline \multirow[b]{2}{*}{28} & \multicolumn{9}{|c|}{ ¿Con qué frecuencia tiene la impresión de que las cosas que hace en su vida diaria son insignificantes? } \\
\hline & Con mucha frecuencia & 1 & 2 & 3 & 4 & 5 & 6 & 7 & Nunca o casi nunca \\
\hline \multirow{2}{*}{29} & \multicolumn{9}{|c|}{ ¿Con qué frecuencia siente que no podrá mantener sus emociones bajo control? } \\
\hline & Con mucha frecuencia & 1 & 2 & 3 & 4 & 5 & 6 & 7 & Nunca o casi nunca \\
\hline
\end{tabular}

